\title{
A direct pedestrian-structure interaction model to characterize the human induced vibrations on slender footbridges
}

\section{Un modelo directo de interacción peatón-estructura para caracterizar las vibraciones inducidas por peatones en pasarelas esbeltas}

\author{
J. F. Jiménez-Alonso $^{(*)}$, A. Sáez ${ }^{(*)}$
}

\section{ABSTRACT}

Although the scientific community had knowledge of the human induced vibration problems in structures since the end of the 19th century, it was not until the occurrence of the vibration phenomenon happened in the Millennium Bridge (London, 2000) that the importance of the problem revealed and a higher level of attention devoted. Despite the large advances achieved in the determination of the human-structure interaction force, one of the main deficiencies of the existing models is the exclusion of the effect of changes in the footbridge dynamic properties due to the presence of pedestrians. In this paper, the formulation of a human-structure interaction model, addresses these limitations, is carried out and its reliability is verified from previously published experimental results.

Keywords: Slender footbridges; human induced vibration; pedestrian-structure interaction; dynamic behaviour change.

\section{RESUMEN}

Aunque la comunidad científica tenía conocimiento de los problemas vibratorios inducidos por peatones en estructuras desde finales del siglo XIX, no fue hasta la ocurrencia de los eventos vibratorios acontecidos en la pasarela del Milenio (Londres, 200o), cuando la importancia del problema se puso de manifiesto y se le comenzó a dedicar un mayor nivel de atención. A pesar de los grandes avances alcanzados en la caracterización de la fuerza de interacción peatón-estructura una de las principales deficiencias de los modelos existentes es la exclusión del cambio en las propiedades dinámicas de la pasarela por la presencia de peatones. En este artículo, se presenta la formulación de un modelo de interacción peatón-estructura que intenta dar respuesta a dichas limitaciones, y su validación a partir de resultados experimentales previamente publicados por otros autores.

Palabras clave: Pasarelas esbeltas; vibraciones inducidas por seres humanos; interacción peatón-estructura; modificación de comportamiento dinámico.

${ }^{(*)}$ University of Seville (España).

Persona de contacto/Corresponding author: jfjimenez@us.es (J. F. Jiménez-Alonso)

Cómo citar este artículo/Citation: Jiménez-Alonso, J. F., Sáez, A. (2014). A direct pedestrian-structure interaction model to characterize the human induced vibrations on slender footbridges. Informes de la Construcción, 66(EXTRA-1): mo07, doi: http://dx.doi.org/10.3989/ic.13.110. Licencia / License: Salvo indicación contraria, todos los contenidos de la edición electrónica de Informes de la Construcción se distribuyen bajo una licencia de uso y distribución Creative Commons Reconocimiento no Comercial 3.o. España (cc-by-nc). 


\section{INTRODUCTION}

The phenomenon of interaction between pedestrians and bridges is known since, at the end of the 19th century (1), a group of 60 soldiers excited, under their step, a bridge located in the British town of Broughton. Although the scientific community did not stop studying this issue, it was the occurrence of the phenomenon happened in the Millennium Bridge (London) that stressed the importance of the problem and led to a higher level of attention (2). In most cases, the effect that the pedestrians induce on the footbridge has been idealized like a moving variable force on the structure (3). The variability of the above mentioned load tries to have in consideration the variation of the level of pressures that takes place between the pedestrian and the deck during the phenomenon of the step. However, in all these models, either the effect that the pedestrians have on the dynamic characteristics of the structure is neglected, or such effect is considered by means of very simplified finger rules. Consequently, these models do not incorporate appropriately the energetic exchange that takes place between both systems during the step of the pedestrian flows on the structure. Nevertheless, in the existing publications (3) there are clear indications about the importance of the dynamic interaction phenomena, with evidence that both the frequencies and the modes of vibration of the structure are affected by the step of pedestrian groups. In the case of structures subjected to large pedestrian flows, the correct estimation of the change of their dynamic properties due to the pedestrian crossing is very important during the design phase, in order to adjust as much as possible the natural frequencies of the structure outside the range of pedestrian step frequencies and, in the case of an intervention on an existing footbridge, in order to improve its comfort level (4) (5).

In the present work, a methodology for the correct characterization of the whole dynamic behavior is proposed, by implementing a human-structure interaction model with three degrees of freedom, in order to characterize the movement of the gravity center of the pedestrian in the three spatial directions. The problem of energetic exchange is addressed in a direct form, realizing the modal projection of the coordinates in contact between the pedestrian and the structure, and maintaining the physical coordinates of the gravity center of the pedestrian. The model considers, in the same way, the local effect of the step by means of the modal projection of the corresponding interaction force.

This procedure of resolution allows, on the one hand, to uncouple the equations of the dynamic system that governs the behavior of the structure, thus facilitating the effective application of the model from the modal characteristics of the footbridge, as obtained from any commercial software based on the finite element method; and on the other hand, it allows to estimate in a direct form both the dynamic characteristics of the structure during the pedestrian step, as well as the components of the pedestrian center of gravity acceleration. Furthermore, additional parameters, such as the sign of pedestrian damping introduced into the system, may be included in the model. Finally, a validation example of the proposed model is presented, where the change of the dynamic behaviour of a real laboratory footbridge during a variable flow of pedestrians is favorably compared with the model predictions.

\section{ANALYSIS OF CURRENT STANDARDS}

Currently, the most advanced international codes about the dynamic behaviour of slender footbridges (4) (5) determine that, in a wide way, if the natural frequencies of the structure is in the range of pedestrian walking step frequency (1.252.30 $\mathrm{Hz}$ for vertical vibrations and $0.50-1.20 \mathrm{~Hz}$ for horizontal vibrations) the acceleration, in that direction, needs to be determined and checked against acceleration limits (Table 1) to guarantee an appropriate comfort level for each design scenario. Furthermore, to avoid lateral synchronization the acceleration in this direction must be below $0.10-0.15 \mathrm{~m} / \mathrm{s}^{2}$.

The design scenario is established by the expected pedestrian traffic (Table 2) and the situation or importance of the structure. The comfort level is determined by the owner of the structure, and normally a medium comfort level must be guaranteed for all traffic classes, except for pedestrian densities above 1.00 $\mathrm{P}$ (Person) $/ \mathrm{m}^{2}$ where a minimum comfort is acceptable.

The pedestrian induced action is represented as an oscillatory distributed load $p(t)$, defined as:

$$
p(t)=G \cdot \cos (2 \cdot \pi \cdot f \cdot t) \cdot n_{p}^{\prime} \cdot \psi
$$

where:

$G$, is the considered component of the step force $(\mathrm{G}=280 \mathrm{~N}$ vertical, $140 \mathrm{~N}$ longitudinal and $35 \mathrm{~N}$ lateral) (4) (5).

$f$, is the natural frequency of the structure under consideration. $n_{p}^{\prime}$, is the equivalent pedestrians number, defined by

[2] $n_{p}^{\prime}=10.80 \cdot \sqrt{\zeta \cdot n_{p}}$ for traffic classes TC1-TC3 or

$$
n_{p}^{\prime}=1.85 \cdot \sqrt{n_{p}} \text { for traffic classes } \mathrm{TC}_{4}-\mathrm{TC}_{5} .
$$

$\psi$, is the reduction coefficient that takes into account the probability that the footfall frequency approaches the natural frequency under consideration.

$\zeta$, is the structural damping ratio.

$n_{p}$, is the number of the pedestrians on the loaded surface $S$ $\left(n_{p}=S \cdot\right.$ density).

$S$, is the loaded surface that depends on the shape of the normal mode under consideration.

Table 1. Defined comfort classes with limit acceleration ranges (5).

\begin{tabular}{|l|l|c|c|}
\hline Level & \multicolumn{1}{|c|}{ Degree } & $\begin{array}{c}\text { Vertical } \\
\text { acceleration }\end{array}$ & $\begin{array}{c}\text { Horizontal } \\
\text { acceleration }\end{array}$ \\
\hline CL1 & Maximum & $<0.50 \mathrm{~m} / \mathrm{s}^{2}$ & $<0.10 \mathrm{~m} / \mathrm{s}^{2}$ \\
\hline $\mathrm{CL} 2$ & Medium & $0.50-1.00 \mathrm{~m} / \mathrm{s}^{2}$ & $0.10-0.30 \mathrm{~m} / \mathrm{s}^{2}$ \\
\hline $\mathrm{CL} 3$ & Minimum & $1.00-2.50 \mathrm{~m} / \mathrm{s}^{2}$ & $0.30-0.80 \mathrm{~m} / \mathrm{s}^{2}$ \\
\hline $\mathrm{CL} 4$ & Discomfort & $>2.50 \mathrm{~m} / \mathrm{s}^{2}$ & $>0.80 \mathrm{~m} / \mathrm{s}^{2}$ \\
\hline
\end{tabular}

Table 2. Traffic classes (5).

\begin{tabular}{|c|c|l|}
\hline Classes & $\begin{array}{c}\text { Density } \\
\boldsymbol{d}\left[\mathbf{P} / \mathbf{m}^{2}\right]\end{array}$ & \multicolumn{1}{|c|}{ Characteristics } \\
\hline $\mathrm{TC} 1$ & $<15 \mathrm{P}$ & 15 single persons \\
\hline $\mathrm{TC} 2$ & $<0.20 \mathrm{P} / \mathrm{m}^{2}$ & Comfortable and free walking \\
\hline $\mathrm{TC}_{3}$ & $<0.50 \mathrm{P} / \mathrm{m}^{2}$ & $\begin{array}{l}\text { Unrestricted walking, significantly } \\
\text { dense traffic }\end{array}$ \\
\hline $\mathrm{TC} 4$ & $<1.00 \mathrm{P} / \mathrm{m}^{2}$ & $\begin{array}{l}\text { Uncomfortable situation, obstructed } \\
\text { walking }\end{array}$ \\
\hline $\mathrm{TC} 5$ & $<1.50 \mathrm{P} / \mathrm{m}^{2}$ & Unpleasant walking, very dense traffic \\
\hline
\end{tabular}


However, this methodology presents some limitations:

- the equivalent pedestrian number (pedestrian moving in phase with the structure) has been determined by the experimental results of only one footbridge (5).

- the change in the dynamic structural properties that the pedestrians flow causes is considered through a finger rule (addition of all the pedestrian mass density to the structure mass matrix).

- the interaction between pedestrians and the structure is only slightly considered, so the international standards do not consider adequately the synchronization phenomenon between pedestrians or between these ones and the structure.

The estimations carried out, under this methodology, normally overestimate the real results (5).

\section{PROPOSAL OF A HUMAN-STRUCTURE INTERACTION MODEL}

In this section a method for the simulation of the interaction between the pedestrian and the footbridge is proposed. It follows from the application of the dynamic equilibrium equations to a simplified model of interaction with sprung and unsprung masses (Figure 1).

For $n$ modes of vibration $\varphi_{i}(x)$, the total response of the structure may be decomposed in terms of the amplitude of the different modes $y_{i}(t)$ as:

$$
w(x, t)=\sum_{i=1}^{n} y_{i}(t) \cdot \varphi_{i}(x)
$$

$$
\dot{w}(x, t)=\sum_{i=1}^{n} \dot{y}_{i}(t) \cdot \varphi_{i}(x)+\sum_{i=1}^{n} y_{i}(t) \cdot v \cdot \varphi_{i}^{\prime}(x)
$$

[6] $\ddot{w}(x, t)=\sum_{i=1}^{n} \ddot{y}_{i}(t) \cdot \varphi_{i}(x)+\sum_{i=1}^{n} 2 \cdot \dot{y}_{i}(t) \cdot v \cdot \varphi_{i}^{\prime}(x)+\sum_{i=1}^{n} y_{i}(t) \cdot v^{2} \cdot \varphi_{i}^{\prime}(x)$ where

[7] and it is neglected, due to its low magnitude, the temporal variation of the step speed $v$.

Considering the equilibrium of the system, structure and pedestrian model, the following coupled equation system may be obtained.

[9]

[10]

$$
M_{i} \ddot{y}_{i}+C_{i} \dot{y}_{i}+K_{i} y_{i}=\varphi_{i}(v t) \cdot F_{\text {int }}
$$

$$
m_{a} \ddot{y}_{a}+c\left(\dot{y}_{a}-\dot{y}_{s}\right)+k\left(y_{a}-y_{s}\right)=0
$$

$$
m_{s} \ddot{y}_{s}+c\left(\dot{y}_{s}-\dot{y}_{a}\right)+k\left(y_{s}-y_{a}\right)=F_{s}-F_{\text {int }}
$$

Thus, $F_{\text {int }}$ follows from the above equation to yield.

$$
F_{\text {int }}=F_{s}-m_{s} \ddot{y}_{s}-c\left(\dot{y}_{s}-\dot{y}_{a}\right)-k\left(y_{s}-y_{a}\right)
$$

And substituting this equation into the equilibrium equation of the structure.

$$
\text { [13] } M_{i} \ddot{y}_{i}+C_{i} \dot{y}_{i}+K_{i} y_{i}=\varphi_{i}(v t) \cdot\left(F_{s}-m_{s} \ddot{y}_{s}-c\left(\dot{y}_{s}-\dot{y}_{a}\right)-k\left(y_{s}-y_{a}\right)\right)
$$

Applying the equations of compatibility of displacements, velocity and acceleration between the structure and the simplified model of interaction.

$$
\begin{aligned}
& y_{s}=w(x, t) \\
& \dot{y}_{s}=\dot{w}(x, t) \\
& \ddot{y}_{s}=\ddot{w}(x, t)
\end{aligned}
$$

Substituting these relations in the overall dynamic equilibrium equation of the structure and organizing information in a matrix form, the following model of interaction is obtained.

[17]

$$
M(t) \cdot \ddot{y}(t)+C(t) \cdot \dot{y}(t)+K(t) \cdot y(t)=F(t)
$$

Considering the nature of the resulting system, the use of a method of $\beta$-Newmark integration family is proposed, with parameters $\beta=1 / 4$ and $\gamma=1 / 2$, thus ensuring an unconditionally stable system.

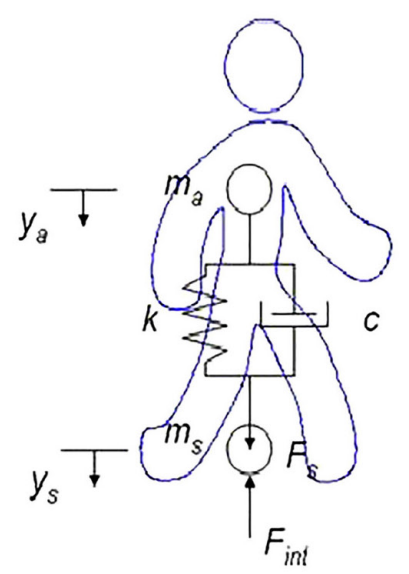

is the spatial derivate of the mode of vibration $i$.

is the second spatial derivate of the mode of vibration $i$.

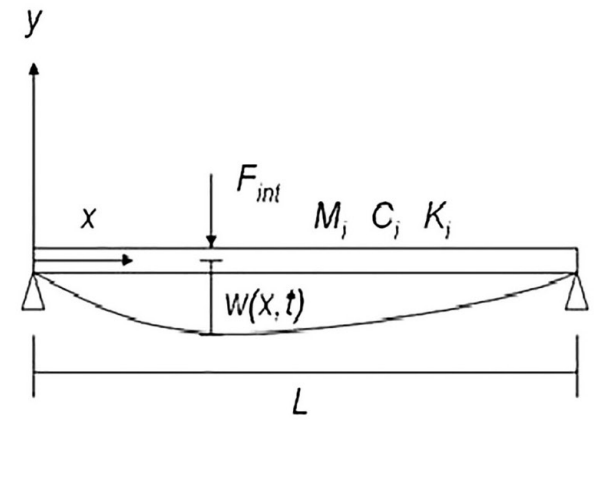

\begin{tabular}{c|l}
$m_{a}$ & sprung mass \\
$m_{s}$ & unsprung mass \\
$m$ & total mass \\
$y_{a}$ & displacement sprung mass \\
$y_{s}$ & displacement unsprung mass \\
$w(x, t)$ & deflection of the structure \\
$k$ & equivalent pedestrian stiffness \\
$c$ & equivalent pedestrian damping \\
$v$ & step speed \\
$F_{i n t}$ & interaction force \\
$F_{s}$ & step force (see section IV) \\
$M_{i}$ & modal mass of the vibration mode i \\
$C_{i}$ & modal damping of the ibration mode i \\
$K_{i}$ & modal stiffness of the vibration mode i \\
$\phi_{i}(x)$ & vibration mode $\mathrm{i}$
\end{tabular}

Figure 1. Pedestrian-structure interaction model. 
In the previous expressions, the value of the vibration modes is zero, when the pedestrian remains outside the structure.

$$
\begin{array}{ll}
\varphi_{i}(x)=0 \text { for } 0 \geq x \geq L & \text { for, with } L \text { being the } \\
\text { length of the structure }
\end{array}
$$

In the proposed method, $\varphi_{i}(x)$ is obtained, in a discrete way, using the finite element method, collecting the modal displacements and derivates in each of the nodes of the structure. To obtain a continuous function of the modes they are determined from the shape functions consistent with the finite element approximation. For the footbridge, the interpolation functions are cubic adopting the Bernoulli hypothesis for the beam elements.

$$
\varphi_{i}(x)=\sum_{j} \varphi_{i}^{j} \cdot N_{j}(x)
$$

Where $N_{j}(x)$ are the shape functions and $\varphi_{i}^{j}$ are the nodal values.

For a group of $k$ pedestrians (Figure 2), we may further represent each one by the above simplified interaction model.

When a group of pedestrians is considered in the calculations, the number of differential equations to solve increases. In the case of a single pedestrian, the proposed model leads to a system of $n+1$ equations, corresponding to the considered number of vibration modes $n$ plus the appropriate simplified interaction mechanical element system. Similarly, when considering a group of $k$ pedestrians, a system of $n+k$ differential equations will need to be solved. It is important to note that the equations for the modes of vibration of the structure vary in terms of the position of pedestrians. At every instant, the numbers of pedestrian on the deformed shape must be calculated, as well as the value of the amplitude, slope and curvature corresponding to their position.

As a preliminary validation of the proposed formulation, the previously defined parameters will be estimated from the results available in the literature for comparable studies (6), as summarized in next sections. Finally, the experimental and numerical dynamic characteristics of a laboratory footbridge, under three controlled group of pedestrians, will be compared in order to determine the parameters and goodness of the proposed model.

The validation will be carried out, for simplicity, in the vertical direction, although the extracted results are easily extrapolated to the other directions.

\section{DETERMINATION OF THE WALKING FORCES}

The movement of the body mass and the put-down, rolling and push-off of the feet of one pedestrian generate the induced three-dimensional forces between both elements, $F$, that according to the research developed by different authors (4), can be determinated from a Fourier series decomposition in the three-space components.

[20]

$$
\begin{gathered}
F_{p, \text { vert }}(t)=P\left[1+\sum_{i=1}^{n_{f}} \alpha_{i, \text { vert }} \sin \left(2 \pi i f_{s} t-\phi_{i}\right)\right] \\
F_{p, \text { lat }}(t)=P \sum_{i=1}^{n_{f}} \alpha_{i, \text { lat }} \sin \left(\pi i f_{s} t-\phi_{i}\right) \\
F_{p, \text { long }}(t)=P \sum_{i=1}^{n_{f}} \alpha_{i, \text { long }} \sin \left(2 \pi i f_{s} t-\phi_{i}\right)
\end{gathered}
$$

where

$F_{p, v e r t}$ vertical periodic force due to walking or running $F_{p, l a t}^{p, v e r t}$ lateral periodic force due to walking or running $F_{p \text {,long }}$ longitudinal periodic force due to walking or running $P[\mathrm{~N}]$ medium pedestrian weight (internationally considered as $P=700.00 \mathrm{~N}$ )

$\alpha_{\mathrm{i}, \text { vert }} \alpha_{\mathrm{i} \text {,lat }} \alpha_{\mathrm{i} \text {,long }}$ Fourier coefficient of the ith harmonic for vertical, lateral and longitudinal forces or dynamic load factor (DLF).

$f_{s}[\mathrm{~Hz}]$ step frequency

$\varphi_{i}$ phase shift of the ith harmonic

$n_{f}$ total number of contributing harmonics.

Among the contributions of the different authors, for the development of the present document, the vertical dynamic

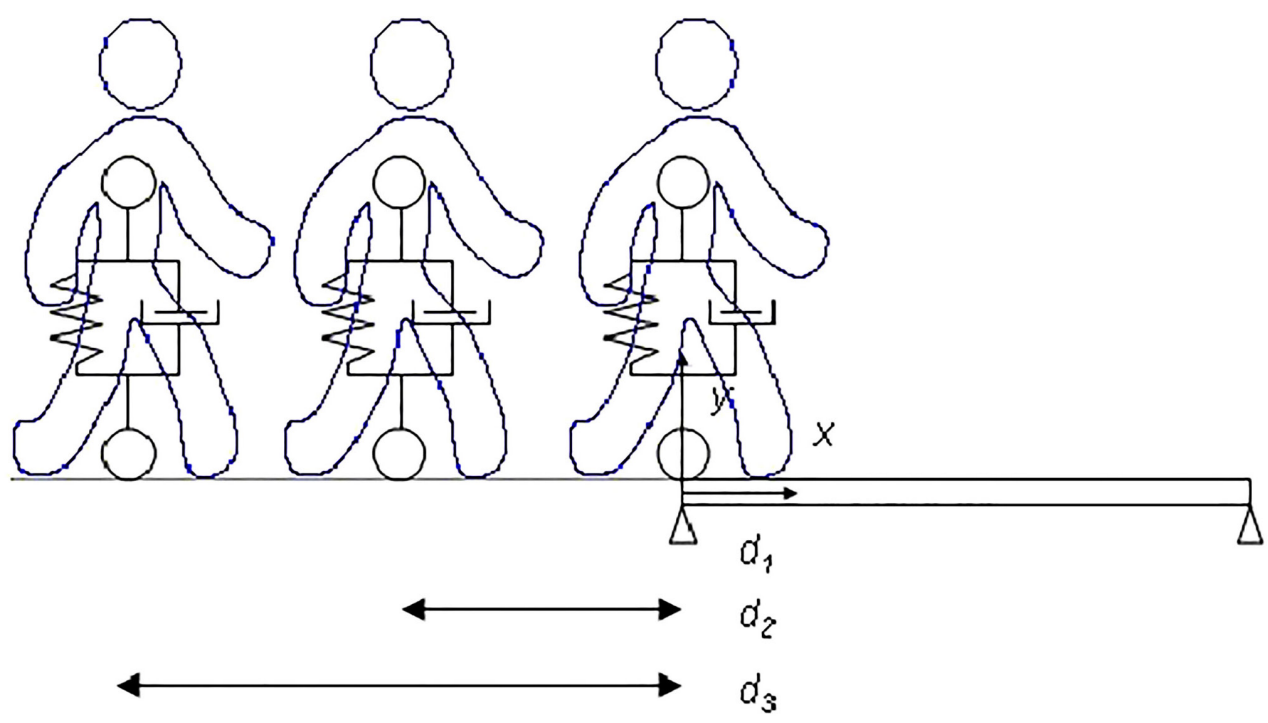

Figure 2. Pedestrians group according to the pedestrian-structure interaction model. 
load factors proposed by Setra (5) (see Table 3 and Figure 3) will be considered to construct and validate our model. This criterion is widely accepted by both the scientific community and the designers of this type of structures. These standards obtained the dynamic coefficients from experimental tests performed on mobile platforms. The pedestrian load, according the results of such tests, is adequately characterized by the contribution of the first three harmonics.

The relation between the velocity magnitude, $v$, and the pacing frequency, $f_{\mathrm{s}}$, is considered by the empirical relationship based on the work of Bertram and Ruina (3).

$$
f_{s}=0.35 \cdot v^{3}-1.59 \cdot v^{2}+2.93 \cdot v
$$

\section{INITIAL ESTIMATION OF THE DYNAMIC PROPERTIES OF THE HUMAN-STRUCTURE INTERACTION MODEL}

For the estimation of the dynamic characteristics of the SDOF-system, as a first approximation, a wide bibliographic study has been made. There are several studies that collect the effect of spectators on stadiums stands in the dynamic behaviour of the structure by a SDOF static system (6). In Figure 4 and Table 4, a scheme of the models used and the estimated dynamic parameters are shown, where $f_{h}$ and $\zeta_{h}$ are the natural frequency and the equivalent damping ratio of the human system.

The above results allow establishing a likely range of variation of the system parameters. Thus, considering the maximum and minimum values (Figure 4 ) of the sprung mass $\left(\mathrm{m}_{\mathrm{a}}\right)$, the equivalent human damping ratio $\left(\zeta_{h}\right)$ and the vertical natural frequency $\left(f_{h}\right)$ of each pedestrian, it is shown in Table 5 a possible range of variation of the parameters of the proposed model. The sprung mass $\left(\mathrm{m}_{\mathrm{a}}\right)$ is presented as a percentage of the total mass. A pedestrian type with a mean total mass of $70.00 \mathrm{~kg}$ is considered, as established by the European standards (4).

Table 3. Fourier coefficient and phase shift for vertical dynamic load factors (5).

\begin{tabular}{|c|c|c|c|c|c|}
\hline $\boldsymbol{\alpha}_{1}$ & $\boldsymbol{\alpha}_{\mathbf{2}}$ & $\boldsymbol{\alpha}_{3}$ & $\boldsymbol{\varphi}_{1}$ & $\boldsymbol{\varphi}_{2}$ & $\boldsymbol{\varphi}$ \\
\hline 0.40 & 0.04 & 0.04 & 0.00 & 90.00 & 90.00 \\
\hline
\end{tabular}
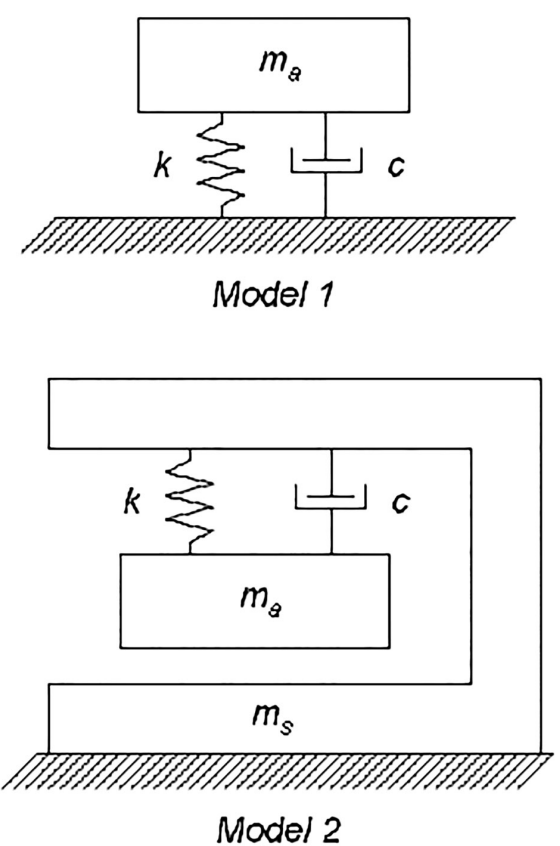

Figure 4. Simplified dynamic representations of the standing human body: (1) SDOF model (2) SDOF model with rigid support.

Table 4. Dynamic properties of SDOF equivalents to standing humans (6).

\begin{tabular}{|c|c|c|c|}
\hline $\begin{array}{c}\text { Human } \\
\text { Model }\end{array}$ & $\begin{array}{c}\text { Modal } \\
\text { Properties }\end{array}$ & $\begin{array}{c}\text { Human } \\
\text { Model }\end{array}$ & $\begin{array}{c}\text { Modal } \\
\text { Properties }\end{array}$ \\
\hline \multirow{3}{*}{$\begin{array}{l}\text { Foschi et al. } \\
\text { (Model 1) }\end{array}$} & $\mathrm{f}_{\mathrm{h}}=3.30 \mathrm{~Hz}$ & \multirow{3}{*}{$\begin{array}{l}\text { Falati } \\
\text { (Model 1) }\end{array}$} & $\mathrm{f}_{\mathrm{h}}=10.43 \mathrm{~Hz}$ \\
\hline & $\zeta_{h}=53.00 \%$ & & $\zeta_{\mathrm{h}}=50.00 \%$ \\
\hline & $\mathrm{m}_{\mathrm{a}}=91.00 \mathrm{~kg}$ & & $\mathrm{~m}_{\mathrm{a}}=25.00 \mathrm{~kg}$ \\
\hline \multirow{3}{*}{$\begin{array}{l}\text { Al-Foqaha'a } \\
\text { (Model 1) }\end{array}$} & $\mathrm{f}_{\mathrm{h}}=3.50 \mathrm{~Hz}$ & \multirow{3}{*}{$\begin{array}{l}\text { Zheng and } \\
\text { Brownjohn } \\
\text { (Model 1) }\end{array}$} & $\mathrm{f}_{\mathrm{h}}=5.24 \mathrm{~Hz}$ \\
\hline & $\zeta_{h}=34.00 \%$ & & $\zeta_{h}=39.00 \%$ \\
\hline & $\mathrm{m}_{\mathrm{a}}=83.00 \mathrm{~kg}$ & & $\mathrm{~m}_{\mathrm{a}}=85.00 \mathrm{~kg}$ \\
\hline \multirow{3}{*}{$\begin{array}{l}\text { Al-Foqaha'a } \\
\text { (Model 2) }\end{array}$} & $f_{h}=3.70 \mathrm{~Hz}$ & \multirow{3}{*}{$\begin{array}{l}\text { Matsumoto } \\
\text { and Griffin } \\
\text { (Model 1) }\end{array}$} & $\mathrm{f}_{\mathrm{h}}=5.74 \mathrm{~Hz}$ \\
\hline & $\zeta_{h}=36.00 \%$ & & $\zeta_{\mathrm{h}}=69.00 \%$ \\
\hline & $\begin{array}{l}\mathrm{m}_{\mathrm{a}}=75.00 \mathrm{~kg} \\
\mathrm{~m}_{\mathrm{s}}=8.00 \mathrm{~kg}\end{array}$ & & $\mathrm{~m}_{\mathrm{a}}=76.10 \mathrm{~kg}$ \\
\hline \multirow{3}{*}{$\begin{array}{l}\text { Brownjohn } \\
\text { (Model 1) }\end{array}$} & $f_{h}=4.90 \mathrm{~Hz}$ & \multirow{3}{*}{$\begin{array}{l}\text { Matsumoto } \\
\text { and Griffin } \\
\text { (Model 2) }\end{array}$} & $\mathrm{f}_{\mathrm{h}}=5.88 \mathrm{~Hz}$ \\
\hline & $\zeta_{h}=37.00 \%$ & & $\zeta_{h}=61.00 \%$ \\
\hline & $\mathrm{m}_{\mathrm{a}}=80.00 \mathrm{~kg}$ & & $\begin{array}{l}\mathrm{m}_{\mathrm{a}}=70.6 \mathrm{okg} \\
\mathrm{m}_{\mathrm{s}}=7.06 \mathrm{~kg}\end{array}$ \\
\hline
\end{tabular}

Wallking Vertical Force

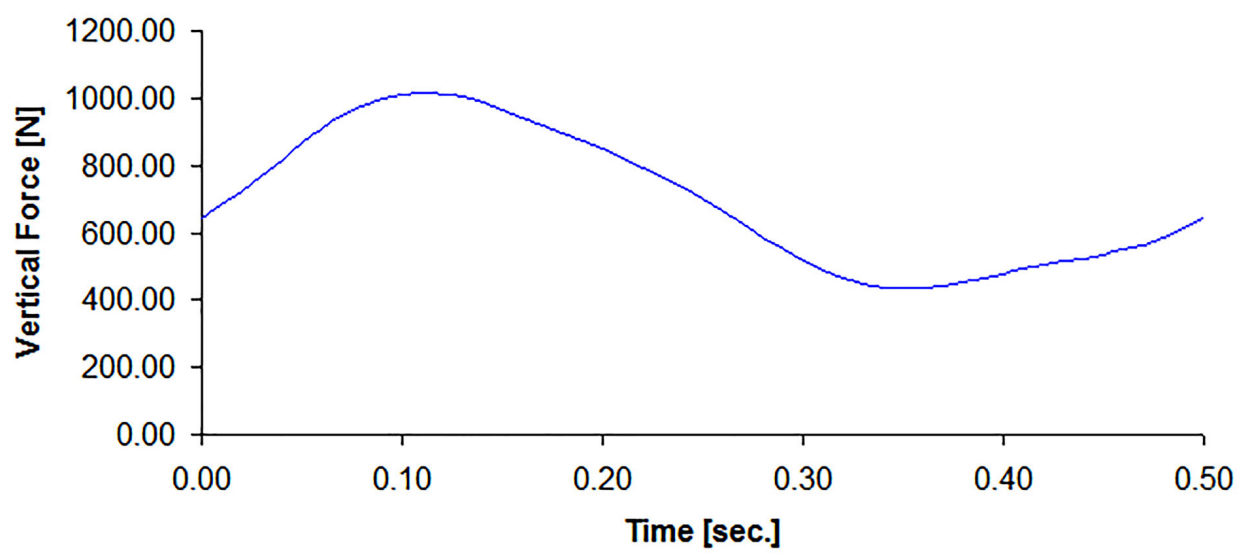

Figure 3. Vertical component of walking pedestrian force (5). 
Table 5. Estimated range of the parameters of the human-structure interaction model.

\begin{tabular}{|l|c|c|c|}
\cline { 2 - 4 } \multicolumn{1}{c|}{} & $\mathbf{m}_{\mathrm{a}}[\%]$ & $\boldsymbol{\zeta}_{\mathrm{h}}[\%]$ & $\mathbf{f}_{\mathrm{h}}[\mathrm{Hz}]$ \\
\hline Minimum & 91.00 & 34.00 & 3.30 \\
\hline Maximum & 100.00 & 69.00 & 10.43 \\
\hline
\end{tabular}

This range of variation is considered as a reference in order to perform a validation of the interaction model in the vertical direction, but provided they are based on a passive pedestrians state, slight variations may be expected.

In order, on one hand, to consider the mass distribution of the human body (3) and on the other hand, the physical understanding of the problem that suggests a reduction of the damping and stiffness of the pedestrian associated with the movement, the above limits are increased, reducing the lower limits to the values set in the following ranges:

- sprung mass, $m_{a}, 80-100 \%$.

- human damping ratio, $\zeta_{h}, 10-69 \%$.

- human natural frequency, $f_{h}, 1.00-10.43 \mathrm{~Hz}$.

The establishment of a search domain improves the efficiency of the parameter identification methods based on a probabilistic search. The value of the parameters of the pedestrian-structure interaction model will be determined using a probabilistic estimation method applied on the above search domain.

\section{EXPERIMENTAL PARAMETERS IDENTIFICATION AND MODEL VALIDATION}

\subsection{Reference experimental results}

Next, we will perform a more efficient estimation of the model parameters and a validation of its reliability by comparing our numerical predictions versus the experimental results obtained by Georgakis and Jorgesen (7). In their work, they studied the change of the dynamic behaviour of a laboratory footbridge due to the step of controlled pedestrians groups.

The laboratory footbridge, considered in (7), is a $16 \mathrm{~m}$-long steel double U simply supported beam. The longitudinal beam profiles are UNP-350, with UNP-200 crossbeams placed at $1400.00 \mathrm{~mm}$ intervals. Several masses were added to the footbridge in different point of the structure to increase the modal mass and thus decrease the footbridge's frequency to a level close to the expected mean step frequency of the pedestrians (Figure 5). The total mass of the structure was $5224.00 \mathrm{~kg}$.

A modal analysis was performed in order to identify the footbridge natural frequencies and damping ratio according to the level of vibration. The footbridge was excited by an actuator in the mid-span point to control its level of displacements. Figures 6 and 7 correlate the dependency between the first vertical vibration frequency $\left(f_{1}\right)$ and the damping ratio $\left(\zeta_{\text {foot }}\right)$ with the footbridge vibration level at mid-span, without taking into account the influence of the pedestrians (7).

Subsequently, the structure was subjected to nine different load scenarios (LS), under a continuous flow of 4, 7 and 10 pedestrians (leading to mean flow rates of approximately $0.35,0.62$ and $0.88 \mathrm{P} / \mathrm{s}$ ), during 180.00 seconds with a step frequency of $1.38 \mathrm{~Hz}$, a step velocity of $1.40 \mathrm{~m} / \mathrm{s}$ and three different mid-span levels of displacement of 1.00, 5.00 and $10.00 \mathrm{~mm}$. Pedestrians were allowed to walk in both directions along the footbridge.

The estimation of the dynamic properties was performed in each case from a frequency sweep of the actuator. The natural a)

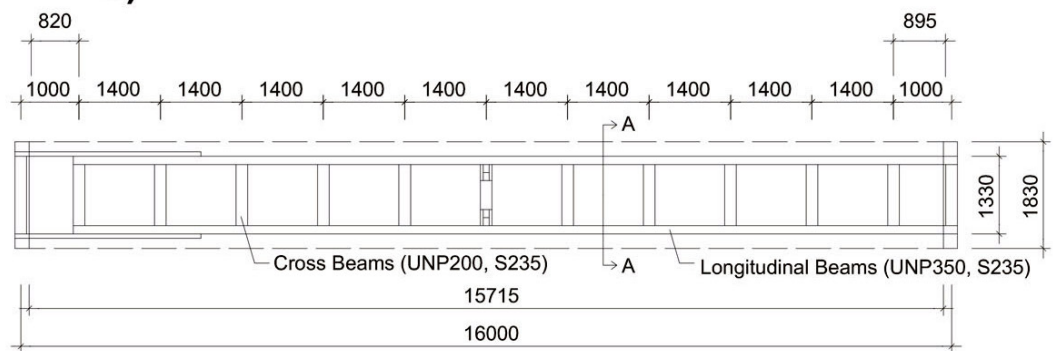

b)

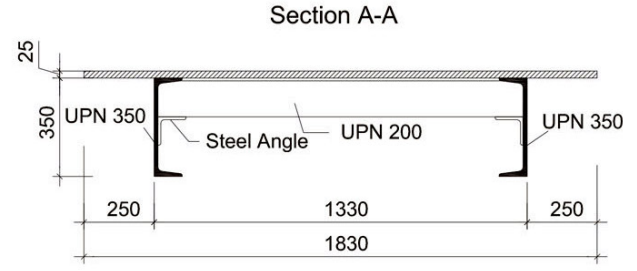

Figure 5. (a) Elevation and (b) cross-section of the laboratory footbridge structure (7).

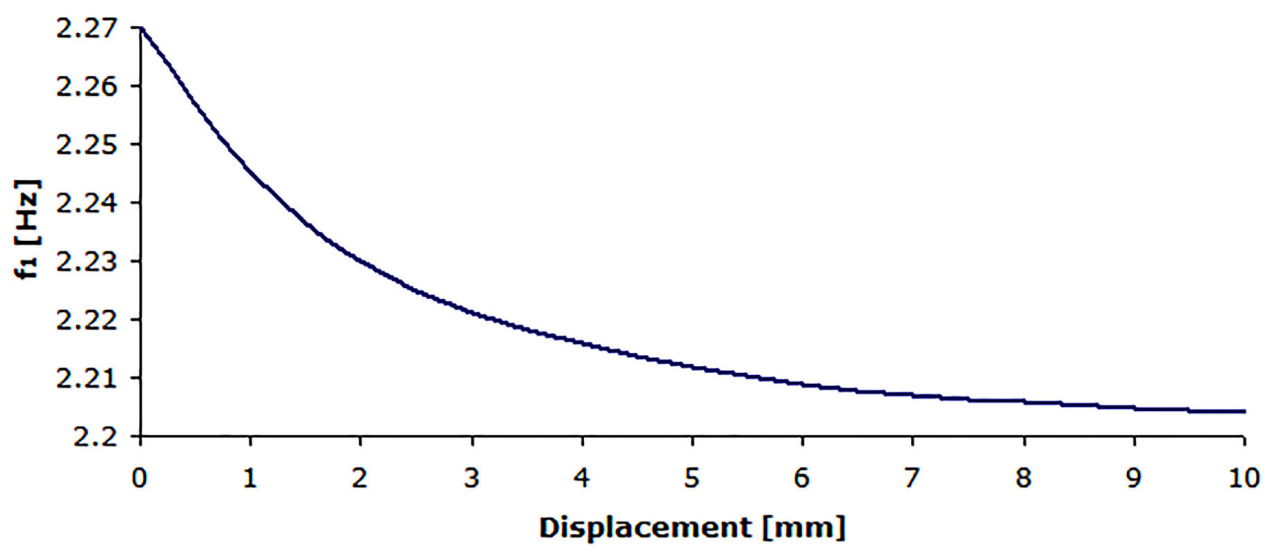

Figure 6. First natural frequency versus vibration amplitude for empty footbridge (7). 


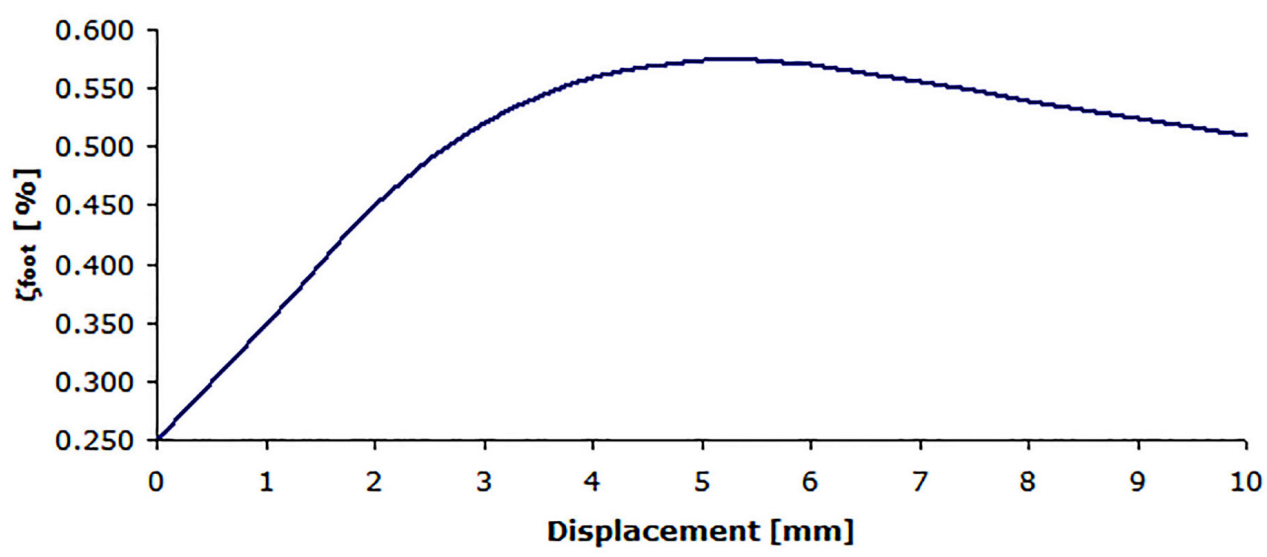

Figure 7. Damping ratio versus vibration amplitude for empty footbridge (7).

frequency was characterized by the frequency where the force exerted by the actuator reaches a minimum value.

The main results of this research, summarized in the Table 6 , show the shift of the first natural frequency of the structure, from $f_{1, \text { emp }}$ (empty footbridge) to $f_{1, \text { full }}$ (full footbridge), versus the level of displacement at the mid-span (A) and the number of pedestrians $\left(N_{p}\right)$.

\subsection{Model validation}

The above results allow obtaining an experimental estimation of the change in the dynamic behaviour of a laboratory footbridge depending on the pedestrian density. From these data, the different parameters of the proposed human-structure interaction model will be estimated by solving the inverse dynamic problem. The solution of this problem will be made in two phases.

First, a finite element model of the footbridge was developed (see Figure 8), using 3-D beam elements and, adjusting an

Table 6. Change of first natural frequency under the different scenarios.

\begin{tabular}{|c|c|c|c|c|}
\hline $\mathbf{L S}$ & $\mathbf{N}_{\mathbf{p}}$ & $\mathbf{A}[\mathbf{m m}]$ & $\mathbf{f}_{\mathbf{1}, \mathbf{e m p}}[\mathbf{H z}]$ & $\mathbf{f}_{\mathbf{1}, \text { full }}[\mathbf{H z}]$ \\
\hline $\mathbf{1 . 1}$ & 4 & 1.00 & 2.280 & 2.086 \\
\hline $\mathbf{1 . 2}$ & 4 & 5.00 & 2.270 & 2.073 \\
\hline $\mathbf{1 . 3}$ & 4 & 10.00 & 2.220 & 2.029 \\
\hline $\mathbf{2 . 1}$ & 7 & 1.00 & 2.280 & 2.022 \\
\hline $\mathbf{2 . 2}$ & 7 & 5.00 & 2.270 & 2.009 \\
\hline $\mathbf{2 . 3}$ & 7 & 10.00 & 2.220 & 1.965 \\
\hline $\mathbf{3 . 1}$ & 10 & 1.00 & 2.300 & 1.980 \\
\hline $\mathbf{3 . 2}$ & 10.00 & 5.00 & 2.290 & 1.970 \\
\hline $\mathbf{3 . 3}$ & 10.00 & 10.00 & 2.270 & 1.950 \\
\hline
\end{tabular}

additional mass distribution so that the value of the first experimental vibration frequency of the footbridge is equal to the one obtained from the numerical modal analysis, considering an empty structure.

Then in order to estimate the three main parameters of the proposed pedestrian-structure interaction model, sprung mass $\left(\mathrm{m}_{\mathrm{a}}\right)$, equivalent human damping ratio $\left(\zeta_{\mathrm{h}}\right)$ and human natural frequency $\left(f_{h}\right)$, the mean square error between the experimental and numerical first vertical natural frequency of the footbridge is minimized (8) for each load scenario (LS). Given the singularity of the minimization function chosen, and in order to avoid local minimums, the genetic algorithms method (a global search method) has been considered (9). The optimization method chosen prevented the solution of the problem from being conditioned by the selected starting point of the search. To avoid ill-conditioning problems, the reduction of the search domain has been developed, constraining the problem. The search range previously proposed has been used.

For each load scenario, a population of 1000 individuals (vectors with three elements) is defined. Each individual modifies through the operations of reproduction, crossover and mutation the values of its elements in order to minimize the value of the objective function. In all the cases, the optimization process reached values of the objective function lower than $10^{-12} \mathrm{~Hz}$. The maximum number of iterations is set to 100 . The numerical value of the damped frequency was obtained from the response function of the system in the frequency domain. The experimental value of the damped frequency was determined for each load scenarios according to the pedestrian flow and the level of vibration reached.

It is shown in Figure 9 the flowchart of the human-structure pedestrian model fitting procedure based on genetic algorithms.

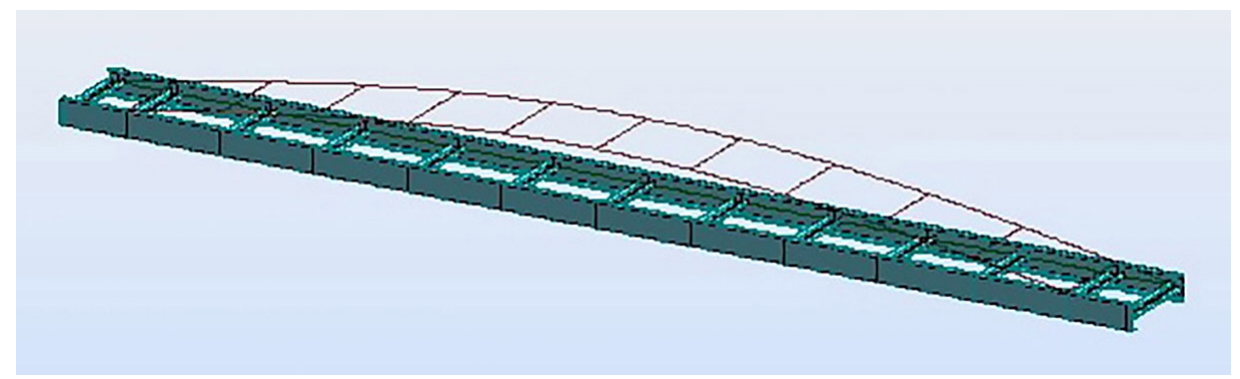

Figure 8. Finite element model and first vibration mode $(\mathrm{f}=2.27 \mathrm{~Hz})$ of the footbridge. 


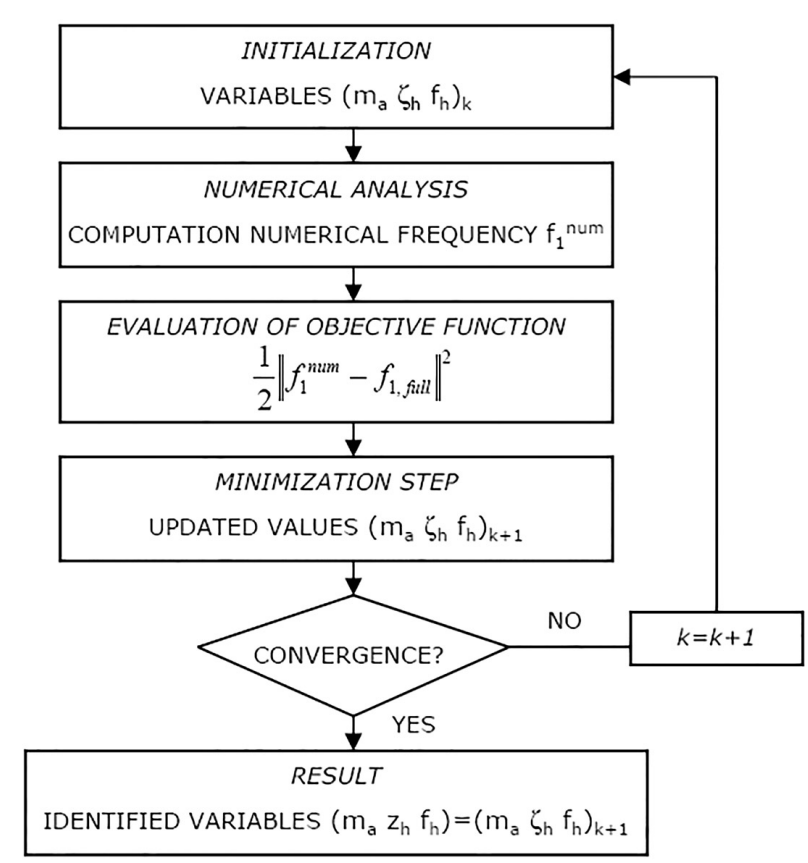

Figure 9. Flowchart of the human-structure pedestrian model fitting procedure.

Table 7. Estimated parameters of the human-structure interaction model.

\begin{tabular}{|c|c|c|c|}
\hline $\mathbf{L S}$ & $\mathbf{m}_{\mathbf{a}}$ [\%] & $\boldsymbol{\zeta}_{\mathbf{h}}$ [\%] & $\mathbf{f}_{\mathbf{h}}$ [Hz] \\
\hline $\mathbf{1 . 1}$ & 83.046 & 45.007 & 2.852 \\
\hline $\mathbf{1 . 2}$ & 84.996 & 45.000 & 2.600 \\
\hline $\mathbf{1 . 3}$ & 84.990 & 45.583 & 2.502 \\
\hline $\mathbf{2 . 1}$ & 84.861 & 43.293 & 2.870 \\
\hline $\mathbf{2 . 2}$ & 84.539 & 49.212 & 2.759 \\
\hline $\mathbf{2 . 3}$ & 84.974 & 49.975 & 2.600 \\
\hline $\mathbf{3 . 1}$ & 82.139 & 48.001 & 2.997 \\
\hline $\mathbf{3 . 2}$ & 83.171 & 51.014 & 2.908 \\
\hline $\mathbf{3 . 3}$ & 83.007 & 47.486 & 2.743 \\
\hline Medium & 83.969 & 47.175 & 2.759 \\
\hline Deviation & 1.054 & 2.611 & 0.165 \\
\hline
\end{tabular}

In Table 7, the results obtained for the nine load scenarios considered are shown. The last two rows of this table show the medium value and the standard deviation obtained for the different parameters. The values of the parameters that characterize the human-structure interaction model remain slightly constant regardless of the level of the pedestrian flow, the level of vibration reached and the damping ratio of the footbridge. The estimated standard deviations of each model parameter (sprung mass, equivalent human damping ratio and natural frequency) are small and the estimated values are considered as appropriate.

In Figure 10, the numerical estimation of the change of the first vertical vibration frequency $\left(f_{1 \text { ver }}\right.$ ) of the structure under the crossing of ten pedestrians is shown. In the figure three curves are represented. The "Experimental Results" shows the change of the experimental first natural frequency according to the results of the modal analysis (7), the "Passive Pedestrians" shows the change of the proposed interaction model with the pedestrian parameters estimated from the medium passive pedestrian behaviour $\left(\mathrm{m}_{\mathrm{a}}=95.5 \%, \zeta_{\mathrm{h}}=51.50 \%\right.$ and $\mathrm{f}_{\mathrm{h}}=6.86 \mathrm{~Hz}$ obtained from Table 5 ), and finally, the "Interaction Model" corresponds to the fitting from the solution of the inverse dynamic problem.

The analysis of the Figure 10 shows that the estimation of first natural frequency of the structure by the proposed model, considering the medium values of the passive pedestrian parameters estimation, produces a relative error of only 5.00 $\%$. Besides the variation of the dynamic properties of the footbridge presents a similar shape to the estimated ones experimentally. Good agreement is observed between the experimental and numerical results.

A comparison of the calculated values with the estimated from the studies on the dynamic effect of passive pedestrians in stadiums, shows mainly a loss of stiffness of each pedestrian when they are in motion.

From the study of the previous results, three main conclusions can be extracted:

- First, the stability that the estimated model parameters present for the different load scenarios, allowing that this model identification may be used as a reliable starting point for the characterization of the effect of the pedestrians on the dynamic behaviour of the structures.

- Then, the appropriately fitting between the experimental and numerical curves that show the change of the first natural frequency of the footbridge, after the adjustment of the pedestrian parameters. The goodness of the correlation verifies the ability of the proposed model to characterize the human-structure interaction problem.

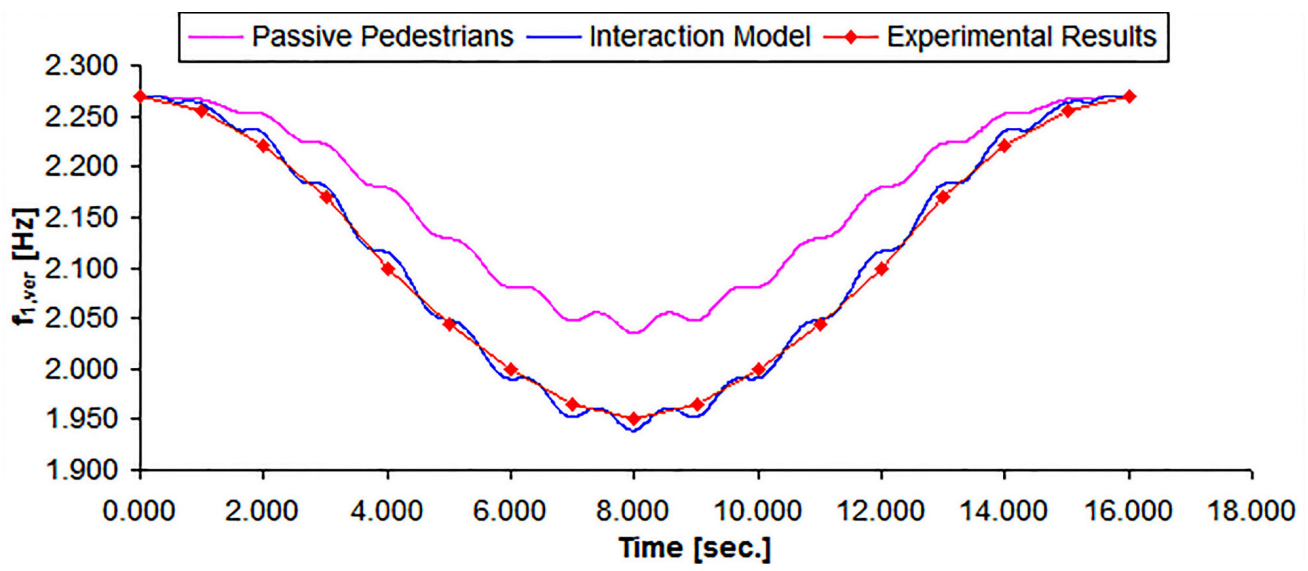

Figure 10. Numerical estimation first natural damped frequency (10 pedestrians). 
- Finally, an important result of the present research is that the main parameter that controls, in the vertical direction, the variation of the natural frequencies of a footbridge under a pedestrian flow is the increase of the damping ratio of the system due to the contribution of the human damping.

\section{CONCLUSIONS}

The models adopted by the current codes for the study of the dynamic behaviour of footbridges do not allow a very accurate estimation of the real response that occurs at the different points of the deck. The most advanced methodology (4) (5), although it is very effective to estimate the sensitivity of the structures to pedestrian flow, it is quite conservative to estimate their effects on the structure. As the acceleration is the main factor that determines the in-service conditions, a pedestrian-structure interaction model has been proposed in order to obtain a more accurate estimation of the response. The model has been presented and its applicability proven in this work. To characterize the accuracy of the presented model, the model parameters are adjusted from the results presented in a scientific study (7) in which the change of the dynamic properties of a laboratory footbridge, under different pedestrian flows, is carried out. The adequate fitting between the experimental and numerical curves that mark the evolution of the first natural frequency of the footbridge under the step of the different group of pedestrians and the stability of the pedestrian parameters for the nine adjustments verify the correct performance of the proposed model to study the problem of human-structure interaction. A medium value, in the vertical direction, for the pedestrian system parameters, is proposed, sprung mass ratio $84.00 \%$, human damping ratio $47.00 \%$ and human frequency $2.75 \mathrm{~Hz}$. However, further studies are needed in order to characterize the dynamic parameters in the three spatial directions under moving situations in real footbridges and furthermore, others factors must be considered in the model in order to include the synchronization phenomena between the pedestrians and the structure.

\section{REFERENCES}

(1) Wolmuth, B., Surtees, J. (2003). Crowd-related failure of bridges. Civil Engineering, 156(3): 116-123.

(2) Dallard, P.,Fitzpatrick, A.J., Le Bourva, S., Low, A., Ridsill Smith, R., Willford, M., Flint, A. (2001). The London Millenium Footbridge. The Structural Engineer, 79(22): 17-33.

(3) Zivanovic, S., Pavic, A., Reynolds, R. (2005). Vibration serviceability of footbridges under human-induced excitation: a literature review. Journal of Sound and Vibration, 279(1-2): 1-74, doi: http://dx.doi.org/10.1016/j.jsv.2004.01.019.

(4) SYNPEX Guidelines. (2007). European Project on Advanced Load Models for synchronous Pedestrian Excitation and Optimised Design Guidelines for Steel Footbridges.

(5) Setra. (2006). Guide méthodologique passerelles piétonnes (Technical guide footbridges: Assement of vibrational behaviour of footbridges under pedestrian loading). Setra.

(6) Jones, C.A., Reynolds, R. , A. Pavic. A. (2011). Vibration serviceability of stadia structures subjected to dynamic crowd loads: A literature review. Journal of Sound and Vibration, 330(8): 1531-1566, doi: http://dx.doi.org/10.1016/j. jsv.2010.10.032.

(7) Georgakis, C., Jorgesen, N.G. (2013). Topics in Dynamics of Bridges, Volume 3. En Proceedings of the 31st IMAC. A Conference on Structural Dynamics, 2013. Chapter 4. Change in Mass and Damping on Vertically Vibrating Footbridges Due to Pedestrians. The Society for Experimental Mechanics, Inc.

(8) Gonzalez, M.N., Cobo, A., Fuente, J.V. (2013). Obtención de modelos de cálculo de sistemas provisionales de protección de borde mediante la técnica de Análisis Modal Operacional. Informes de la Construcción, 65(529): 99-106, doi: http:// dx.doi.org/10.3989/ic.11.133.

(9) Ghee, C., Perry, M.J. (2010). Structural Identification and Damage Detection using Genetic Algorithms: Structures and Infrastructures Book Series, Vol. 6. London: CRC Press 2010. 